$12-1-2008$

\title{
Scattering of an Electromagnetic Plane Wave by a Luneburg Lens. III. Finely Stratified Sphere Model
}

James A. Lock

Cleveland State University, j.lock@csuohio.edu

Follow this and additional works at: https://engagedscholarship.csuohio.edu/sciphysics_facpub

Part of the Physics Commons

How does access to this work benefit you? Let us know!

Publisher's Statement

This paper was published in Journal of the Optical Society of America A: Optics Image Science and Vision and is made available as an electronic reprint with the permission of OSA. The paper can be found at the following URL on the OSA website: http://www.opticsinfobase.org/josaa/ abstract.cfm?URI=josaa-25-12-2991. Systematic or multiple reproduction or distribution to multiple locations via electronic or other means is prohibited and is subject to penalties under law.

\section{Original Citation}

Lock, James A. "Scattering of an Electromagnetic Plane Wave by a Luneburg Lens. III. Finely Stratified Sphere Model." Journal of the Optical Society of America A: Optics Image Science and Vision 25 (2008): 2991-3000.

\section{Repository Citation}

Lock, James A., "Scattering of an Electromagnetic Plane Wave by a Luneburg Lens. III. Finely Stratified Sphere Model" (2008). Physics Faculty Publications. 32.

https://engagedscholarship.csuohio.edu/sciphysics_facpub/32

This Article is brought to you for free and open access by the Physics Department at EngagedScholarship@CSU. It has been accepted for inclusion in Physics Faculty Publications by an authorized administrator of EngagedScholarship@CSU. For more information, please contact library.es@csuohio.edu. 


\title{
Scattering of an electromagnetic plane wave by a Luneburg lens. III. Finely stratified sphere model
}

\author{
James A. Lock \\ Department of Physics, Cleveland State University, Cleveland, Ohio 44115, USA (j.lock@Csuohio.edu)
}

Received July 30, 2008; accepted September 11, 2008;

posted September 29, 2008 (Doc. ID 99459); published November 12, 2008

\begin{abstract}
The parallel iteration procedure for computing scattering by a multilayer sphere is described. The procedure uses a successive doubling strategy applied to four sets of multiple-scattering amplitudes, which is reminiscent of the fast Fourier transform (FFT) algorithm. The procedure is then used to calculate scattering of a plane wave by a modified Luneburg lens. The evolution of the transmission rainbow for the Luneburg lens parameter $f>1$ into an orbiting ray for $f=1$ and into a series of morphology-dependent resonances for $f<1$ is studied, and various features of the scattered intensity as a function of scattering angle are commented on. It is found that some resonances are formed without the presence of an exterior centrifugal barrier to confine them. (C) 2008 Optical Society of America

OCIS codes: $080.2710,260.5740,290.4020$.
\end{abstract}

\section{INTRODUCTION}

This is the third and final paper in a series that examines scattering of a plane wave by a sphere whose refractive index profile is that of either a Luneburg lens or a modified Luneburg lens. This example of electromagnetic scattering by a radially inhomogeneous sphere is both rich in scattering phenomena and simple in that the analysis of many of the phenomena in ray theory is analytically exactly soluble. In [1], transmission through the sphere was considered in ray theory, and arguments were outlined suggesting that both external reflection from the surface and transmission following a number of internal reflections from the surface vanish in the $\lambda \rightarrow 0$ limit. In [2], the transverse electric (TE) polarization was examined semiquantitatively in wave theory by analyzing the effective radial potential of a partial wave, and an explanation was given as to why the transverse magnetic (TM) polarization is not amenable to such an analysis. In this paper all scattering processes are considered in wave theory for both polarizations. Rather than numerically computing the exact interior partial wave radial functions for the inhomogeneous sphere and then using them to obtain the partial wave scattering amplitudes, the modified Luneburg lens is instead approximated by a finely stratified multilayer sphere, and the multilayer sphere scattering problem is solved numerically.

There are two procedures for computing the partial wave scattering amplitudes of a multilayer sphere: (i) the progressive iteration procedure [3-9] and (ii) the parallel iteration procedure [10]. The progressive procedure is valid for any number of layers $M$. One starts by calculating single-scattering partial wave amplitudes at the core, then iteratively progressing outward toward the sphere surface, adding on one layer at a time and recalculating the amplitudes. But in doing this, Riccati-Bessel functions and Riccati-Neumann functions must be evaluated for small arguments comparable to the core size and large partial wave numbers comparable to the overall sphere size. Such computations are prone to numerical overflow and underflow problems, especially when these results are combined over and over again as the iteration progresses outward toward the sphere surface [3]. In spite of these potential numerical difficulties, stable and highly accurate progressive iteration computer programs have been written [3-9] that compute scattering by a multilayer sphere, carefully avoiding the overflow and underflow problems.

On the other hand, the parallel iteration procedure starts by calculating four single-scattering amplitudes at every interface of the multilayer sphere, assuming the number of layers is $M=2^{P}$. These four amplitudes are then combined together at pairs of adjacent interfaces from the core of the sphere to its surface [10]. Adjacent pairs of the four new amplitudes are again combined together iteratively until the four amplitudes for the entire sphere are obtained. This combination at pairs of interfaces performed in parallel from the core to the surface is reminiscent of the successive doubling strategy of the fast Fourier transform (FFT) algorithm [11]. Both the progressive and parallel iteration procedures require $M$ combinations of each scattering amplitude. The progressive procedure uses the innermost part of the sphere for more combinations than the outermost part, whereas the parallel procedure uses all parts of the sphere equally in combinations. Although parallel iteration cannot be applied to such relatively simple systems as $M=3$ or $M=5$, it provides a robust and efficient alternative to progressive iteration when a sphere with a radially inhomogeneous refractive index profile is modeled as a finely stratified multilayer sphere having $M=2^{P}$ layers.

The body of this paper is organized as follows. In Section 2 the geometry is summarized. In Section 3 the de- 
tails of the parallel iteration procedure are given, and the way in which it avoids potential numerical overflow and underflow difficulties is described. In Section 4 numerical results are presented and interpreted for (i) the intensity as a function of scattering angle for various values of the modified Luneburg lens parameter $f$, and (ii) the size parameters of morphology-dependent resonances (MDRs) that occur for $f<1$. In each case special note is made to the ways in which Luneburg lens scattering differs from that of a homogeneous sphere. Finally, in Section 5 a few general conclusions are presented.

\section{GEOMETRY AND NOTATION}

Consider a sphere of radius $a$ centered at the origin of coordinates and composed of $M$ concentric layers of equal width $\Delta=a / M$. An individual layer is denoted by $j$, where $1 \leqslant j \leqslant M$. The core is layer 1 , the outermost layer is $M$, and the medium exterior to the sphere is layer $M+1$. The radius of layer $j$ is $a_{j}=j \Delta$, and the overall sphere radius is $a_{M}=a$. The continuous refractive index profile $N(r)$ of a radially inhomogeneous sphere is discretized to a constant refractive index $N_{j}=N\left(a_{j}-\Delta / 2\right)$ in each of the layers, and $N_{j}$ is assumed in this paper to be real. The interface between layers $j$ and $j+1$ is called the $j, j+1$ interface. The free-space wavelength of the electromagnetic plane wave incident on the multilayer sphere is $\lambda$, the wave number is $k=2 \pi / \lambda$, and the exterior and interior size parameters of the $j, j+1$ interface are

$$
\begin{aligned}
& X_{j, j+1}=N_{j+1} k a_{j}, \\
& Y_{j, j+1}=N_{j} k a_{j} .
\end{aligned}
$$

respectively. The refractive index profile of a modified Luneburg lens is

$$
N(r)=\left[1+f^{2}-(r / a)^{2}\right]^{1 / 2} / f
$$

for $r \leqslant a$.

\section{PARALLEL ITERATION PROCEDURE}

A. Factorization of the Multilayer Sphere Amplitudes In the parallel iteration procedure for calculating scattering by a multilayer sphere [10], four basic partial wave amplitudes $N_{n}, D_{n}, P_{n}$, and $Q_{n}$ for each partial wave number $n$ are used to construct the partial wave scattering amplitudes $a_{n}$ and $b_{n}$. In order to avoid overflow and underflow problems, these amplitudes are factored into terms that rapidly increase or decrease as $n$ increases and terms that remain relatively well behaved. When $a_{n}$ and $b_{n}$ are finally obtained in the last step of the procedure, all the rapidly increasing and decreasing factors are found to cancel, leaving only ratios of the well-behaved terms. Thus only the well-behaved terms need be numerically computed. Once the partial wave scattering amplitudes are obtained, the scattered intensity is computed in the standard way [12]. It should be noted that this cancellation of the rapidly increasing and decreasing factors does not occur for the interior amplitudes $c_{n}$ and $d_{n}$.

As a first step of the parallel iteration procedure, three functions that appear naturally in the factorization of the amplitudes are formed from ratios of Riccati-Bessel functions $\psi_{n}(w)$ and Riccati-Neumann functions $\chi_{n}(w)$,

$$
\begin{aligned}
& E_{n}(w)=\psi_{n}^{\prime}(w) / \psi_{n}(w), \\
& F_{n}(w)=\chi_{n}^{\prime}(w) / \chi_{n}(w), \\
& G_{n}(w)=\psi_{n}(w) / \chi_{n}(w),
\end{aligned}
$$

where the prime symbol indicates a derivative with respect to the argument of the function. For small $w$ corresponding to the radius of a layer near the core and a large partial wave $n$ corresponding to the overall sphere radius, these functions have the asymptotic behaviors [13]

$$
\begin{gathered}
E_{n}(w) \rightarrow(n+1) / w, \\
F_{n}(w) \rightarrow-n / w, \\
G_{n}(w) \rightarrow\left(-\frac{1}{2}\right)(e / 2)^{2 n+1}[w /(n+1 / 2)]^{2 n+1} .
\end{gathered}
$$

As $n \gg w$, both $E_{n}$ and $F_{n}$ only slowly increase, but $G_{n}$ rapidly decreases. However, the ratio

$$
G_{n}(w) / G_{n}(w+\varepsilon) \rightarrow(1-\varepsilon / w)^{2 n+1},
$$

which appears in the factorization of the amplitudes, slowly decreases as long as $\varepsilon<w$.

Following [10], the factorization of the amplitudes used to construct $a_{n}$ and $b_{n}$ proceeds in four steps. (i) First, four basic single-scattering amplitudes are calculated at each interface from the core of the multilayer sphere to its outer surface. (ii) Next, one combines the amplitudes of two adjacent interfaces together to form four basic multiple-scattering amplitudes for each of the adjacent interface pairs from the core to the surface. (iii) This procedure of combining together the four basic amplitudes at pairs of adjacent clusters of interfaces is repeated until one obtains a single set of the four basic multiplescattering amplitudes for the entire $M=2^{P}$ layer sphere. (iv) Finally, two of the final four amplitudes are used to form the partial wave scattering amplitudes $a_{n}$ and $b_{n}$. For notational simplicity, in the remainder of this section the partial wave number is omitted and the TE or TM polarization state is implicit in the values of $\alpha$ and $\beta$, which are defined as

$$
\begin{aligned}
\alpha & =N_{j} \quad \text { for } \mathrm{TE}, \\
& =N_{j+1} \quad \text { for } \mathrm{TM}, \\
& =N_{j+1} \quad \text { for } \mathrm{TE}, \\
& =N_{j} \quad \text { for } \mathrm{TM} .
\end{aligned}
$$

The following development switches back and forth among the specific examples of the homogeneous sphere, the coated sphere, etc., and the totally general case of the $M$ layer sphere with an arbitrary refractive index profile. The four basic single-scattering amplitudes for the $j, j+1$ interface are [10]

$$
N_{j, j+1}=\alpha \psi\left(X_{j, j+1}\right) \psi^{\prime}\left(Y_{j, j+1}\right)-\beta \psi^{\prime}\left(X_{j, j+1}\right) \psi\left(Y_{j, j+1}\right),
$$




$$
\begin{gathered}
D_{j, j+1}=\alpha \chi\left(X_{j, j+1}\right) \psi^{\prime}\left(Y_{j, j+1}\right)-\beta \chi^{\prime}\left(X_{j, j+1}\right) \psi\left(Y_{j, j+1}\right), \\
P_{j, j+1}=\alpha \psi\left(X_{j, j+1}\right) \chi^{\prime}\left(Y_{j, j+1}\right)-\beta \psi^{\prime}\left(X_{j, j+1}\right) \chi\left(Y_{j, j+1}\right), \\
Q_{j, j+1}=\alpha \chi\left(X_{j, j+1}\right) \chi^{\prime}\left(Y_{j, j+1}\right)-\beta \chi^{\prime}\left(X_{j, j+1}\right) \chi\left(Y_{j, j+1}\right) .
\end{gathered}
$$

Equations (9a)-(9d) can be factored as

$$
\begin{aligned}
& N_{j, j+1}=\chi\left(X_{j, j+1}\right) \chi\left(Y_{j, j+1}\right) G\left(X_{j, j+1}\right) G\left(Y_{j, j+1}\right) n_{j, j+1}, \\
& D_{j, j+1}=\chi\left(X_{j, j+1}\right) \chi\left(Y_{j, j+1}\right) G\left(Y_{j, j+1}\right) d_{j, j+1} \\
& P_{j, j+1}=\chi\left(X_{j, j+1}\right) \chi\left(Y_{j, j+1}\right) G\left(X_{j, j+1}\right) p_{j, j+1} \\
& Q_{j, j+1}=\chi\left(X_{j, j+1}\right) \chi\left(Y_{j, j+1}\right) q_{j, j+1}
\end{aligned}
$$

where

$$
\begin{aligned}
& n_{j, j+1}=\alpha E\left(Y_{j, j+1}\right)-\beta E\left(X_{j, j+1}\right), \\
& d_{j, j+1}=\alpha E\left(Y_{j, j+1}\right)-\beta F\left(X_{j, j+1}\right), \\
& p_{j, j+1}=\alpha F\left(Y_{j, j+1}\right)-\beta E\left(X_{j, j+1}\right), \\
& q_{j, j+1}=\alpha F\left(Y_{j, j+1}\right)-\beta F\left(X_{j, j+1}\right) .
\end{aligned}
$$

The terms $\chi\left(X_{j, j+1}\right) \chi\left(Y_{j, j+1}\right)$ can be considered as common factors in Eqs. (10a)-(10d) and at the end of the calculation will cancel. Thus only $n_{j, j+1}, d_{j, j+1}, p_{j, j+1}, q_{j, j+1}$, and some of the $G$ factors need be computed. As an example of this cancellation, a homogeneous sphere has $M=1, j=1$, and the partial wave scattering amplitudes are

$$
a_{n}, b_{n}=N_{12} /\left(N_{12}+i D_{12}\right) .
$$

Substituting Eqs. (10a)-(10d) into Eq. (12), the factors $\chi\left(X_{12}\right) \chi\left(Y_{12}\right)$ cancel, as also does $G\left(Y_{12}\right)$, and one has

$$
a_{n}, b_{n}=G\left(X_{12}\right) n_{12} /\left[G\left(X_{12}\right) n_{12}+i d_{12}\right] .
$$

Proceeding to the next step, for the specific case of a coated sphere with $M=2$ and $j=1,2$, the four basic multiple-scattering amplitudes $N_{123}, D_{123}, P_{123}, Q_{123}$ are obtained [10] from combinations of the four basic singlescattering amplitudes $N_{12}, D_{12}, P_{12}, Q_{12}$ at interface 1,2 and $N_{23}, D_{23}, P_{23}, Q_{23}$ at interface 2,3. One has

$$
\begin{aligned}
& N_{123}=D_{12} N_{23}-N_{12} P_{23}=G\left(Y_{12}\right) G\left(Y_{23}\right) G\left(X_{23}\right) n_{123}, \\
& D_{123}=D_{12} D_{23}-N_{12} Q_{23}=G\left(Y_{12}\right) G\left(Y_{23}\right) d_{123}, \\
& P_{123}=Q_{12} N_{23}-P_{12} P_{23}=G\left(Y_{23}\right) G\left(X_{23}\right) p_{123}, \\
& Q_{123}=Q_{12} D_{23}-P_{12} Q_{23}=G\left(Y_{23}\right) q_{123},
\end{aligned}
$$

where

$$
\begin{aligned}
& n_{123}=d_{12} n_{23}-n_{12} p_{23} G\left(X_{12}\right) / G\left(Y_{23}\right), \\
& d_{123}=d_{12} d_{23}-n_{12} q_{23} G\left(X_{12}\right) / G\left(Y_{23}\right), \\
& p_{123}=q_{12} n_{23}-p_{12} p_{23} G\left(X_{12}\right) / G\left(Y_{23}\right),
\end{aligned}
$$

$$
q_{123}=q_{12} d_{23}-p_{12} q_{23} G\left(X_{12}\right) / G\left(Y_{23}\right) .
$$

The term $G\left(Y_{23}\right)$ again can be considered as a common factor in Eqs. (14a)-(14d) and at the end of the calculation will cancel. The partial wave scattering amplitudes are

$$
a_{n}, b_{n}=N_{123} /\left(N_{123}+i D_{123}\right) \text {. }
$$

Substituting Eqs. (14a)-(14d) into Eq. (16), the factor $G\left(Y_{12}\right)$ cancels as well, and the coated sphere partial wave scattering amplitudes become

$$
a_{n}, b_{n}=G\left(X_{23}\right) n_{123} /\left[G\left(X_{23}\right) n_{123}+i d_{123}\right] .
$$

Again, only $n_{123}, d_{123}, p_{123}, q_{123}$, and $G\left(X_{23}\right)$ need be calculated. For the case of a general multilayer sphere, the multiple-scattering amplitudes $N_{j-1, j, j+1}, \quad D_{j-1, j, j+1}$, $P_{j-1, j, j+1}, Q_{j-1, j, j+1}$ for the combination of the $j-1, j$ and $j, j+1$ interfaces analogous to Eqs. (14a)-(14d) are obtained in an identical way from combinations of the single-scattering amplitudes $N_{j-1, j}, D_{j-1, j}, P_{j-1, j}, Q_{j-1, j}$ at interface $j-1, j$, and $N_{j, j+1}, D_{j, j+1}, P_{j, j+1}, Q_{j, j+1}$ at interface $j, j+1$. There are now half as many amplitudes as there were in the previous step, and the term $G\left(Y_{j, j+1}\right)$ can be considered as a common factor to $N_{j-1, j, j+1}, D_{j-1, j, j+1}$, $P_{j-1, j, j+1}, Q_{j-1, j, j+1}$ that will cancel at the end of the calculation.

In like manner, pairs of adjacent two-interface amplitudes can be combined together in parallel from the core of the sphere to the surface to form four-interface amplitudes in exactly the same way as pairs of adjacent singleinterface amplitudes were combined in Eqs. (14a)-(14d) to form two-interface amplitudes. There are again half as many of these as there were in the previous step, and common factors of $G(Y)$ will cancel at the end of the calculation. Adjacent pairs of these can be combined together in a generalization of Eqs. (14a)-(14d) in parallel from the core to the surface to form eight-interface amplitudes with common $G(Y)$ factors that will cancel, etc. This procedure is continued until one set of composite partial wave amplitudes $N_{12 \ldots M+1}, D_{12 \ldots M+1}, P_{12 \ldots M+1}, Q_{12 \ldots M+1}$ for the entire $M=2^{P}$ layer sphere have been formed. They are factored into a large number of rapidly varying $G(Y)$ terms and the well-behaved terms $n_{12 \ldots M+1}, d_{12 \ldots M+1}$, $p_{12 \ldots M+1}, q_{12 \ldots M+1}$. The partial wave scattering amplitudes for scattering by the entire multilayer sphere are [10]

$$
a_{n}, b_{n}=N_{12 \ldots M+1} /\left(N_{12 \ldots M+1}+i D_{12 \ldots M+1}\right) .
$$

Substituting in, the rapidly varying terms cancel, and one obtains

$$
a_{n}, b_{n}=G\left(X_{M, M+1}\right) n_{12 \ldots M+1} /\left[G\left(X_{M, M+1}\right) n_{12 \ldots M+1}+i d_{12 \ldots M+1}\right] .
$$

Thus only $n_{12 \ldots M+1}, \quad d_{12 \ldots M+1}, \quad p_{12 \ldots M+1}, \quad q_{12 \ldots M+1}, \quad$ and $G\left(X_{M, M+1}\right)$ need be computed. This procedure is valid for any refractive index profile $N(r) \rightarrow N_{j}$ with $1 \leqslant j \leqslant 2^{P}$.

Equations (9)-(19) warrant a number of comments concerning the way in which the factorization process avoids numerical overflow and underflow problems. These comments are perhaps best illustrated in terms of a specific example. Consider an $M=128$ layer sphere of radius $a$ $=5.12 \mu \mathrm{m}$ illuminated by light with wavelength $\lambda$ $=0.512 \mu \mathrm{m}$. The thickness of each layer is $\Delta=0.04 \mu \mathrm{m}$. 
The sphere is assumed to be a Luneburg lens with $f=1.0$ and $N(0)=1.414$, as in Eq. (2). The overall size parameter of the sphere is $X_{128,129}=62.83$. The convergence of the partial wave sum as $n \rightarrow \infty$ is determined by the simultaneous faster-than-exponential increase in $\chi_{n}(k a)$ in the denominator of $a_{n}$ and $b_{n}$ and the faster-than-exponential decrease in $\psi_{n}(k a)$ in the numerator, independent of the interior functions $F_{n}(k a)$ and $G_{n}(k a)$ of [2]. This is because the second set of interior functions $U_{n}(k a)$ and $V_{n}(k a)$ of [2] do not occur in $a_{n}$ and $b_{n}$. Thus the largest partial wave $n_{\max }$ of the sum required for single-precision accuracy is

$$
n_{\max }=1+X_{128,129}+4.3\left(X_{128,129}\right)^{1 / 3}=80 .
$$

The combination of the smallest argument $X_{12}=0.694$ and largest partial wave number $n=80$ gives [13] $\psi_{80}(0.694)$ $\approx 10^{-157}$ and $\chi_{80}(0.694) \approx 10^{155}$. But the individual singleinterface amplitudes of Eqs. (11a)-(11d) contain only the functions $E$ and $F$, which never increase beyond $10^{2}$ for this example. Similarly, $G_{80}(0.694) \approx 10^{-322}$. But since only ratios of the $G$ functions appear in Eqs. (15a)-(15d), the smallest ratio is $G_{80}\left(X_{12}\right) / G_{80}\left(Y_{23}\right) \approx 10^{-49}$, which is more manageable. Finally, since $X_{128,129}$ is the overall size parameter of the sphere, $\psi_{n}\left(X_{128,129}\right)$ and $\chi_{n}\left(X_{128,129}\right)$ are computed using the usual procedures of double-precision upward recursion and downward recursion [14], respectively, to form $G\left(X_{128,129}\right)$ in Eq. (19).

\section{B. Numerical Evaluation of $E_{n}(w), F_{n}(w)$, and $G_{n}(w) / G_{n}(v)$}

The function $E_{n}(w)$ is the logarithmic derivative of $\psi_{n}(w)$, and there are widely publicized routines for computing this for a real refractive index, as assumed here, using downward recursion $[6,15]$. For example,

$$
E_{n-1}(w)=(n / w)-1 /\left[E_{n}(w)+(n / w)\right]
$$

for $l \leqslant n \leqslant n_{\text {start }}$ with

$$
\begin{gathered}
n_{\text {start }}=n_{\max }+15 \\
E_{n_{\mathrm{start}}+1}(w)=0 .
\end{gathered}
$$

The function $F_{n}(w)$ is found to be stable when computed using upward recursion. Starting with

$$
F_{0}(w)=-\tan (w)
$$

the upward recursion is

$$
F_{n}(w)=-(n / w)+\left[(n / w)-F_{n-1}(w)\right] .
$$

The ratio $G_{n}\left(X_{j-1, j}\right) / G_{n}\left(Y_{j, j+1}\right)$ is also found to be stable when computed using upward recursion, assuming that $E_{n}\left(X_{j-1, j}\right), E_{n}\left(Y_{j, j+1}\right), F_{n}\left(X_{j-1, j}\right)$, and $F_{n}\left(Y_{j, j+1}\right)$ have already been separately computed. Starting with

$$
G_{0}\left(X_{j-1, j}\right) / G_{0}\left(Y_{j, j+1}\right)=\tan \left(X_{j-1, j}\right) / \tan \left(Y_{j, j+1}\right),
$$

the upward recursion is

$$
\begin{aligned}
G_{n}\left(X_{j-1, j}\right) / G_{n}\left(Y_{j, j+1}\right)= & G_{n-1}\left(X_{j-1, j}\right)\left[\left(n / X_{j-1, j}\right)+F_{n}\left(X_{j-1, j}\right)\right] \\
& \times\left[\left(n / Y_{j, j+1}\right)+E_{n}\left(Y_{j, j+1}\right)\right] /\left\{G_{n-1}\left(Y_{j, j+1}\right)\right. \\
& \times\left[\left(n / X_{j-1, j}\right)+E_{n}\left(X_{j-1, j}\right)\right]\left[\left(n / Y_{j, j+1}\right)\right. \\
& \left.\left.+F_{n}\left(Y_{j, j+1}\right)\right]\right\} .
\end{aligned}
$$

The ratio of the $G$ functions appearing in the generalization of Eqs. (15a)-(15d) is calculated for the following values of $j$. Let $T$ be the iteration number for the $M=2^{P}$ layer sphere, where $1 \leqslant T \leqslant P-1$, and let $K$ be an integer such that $0 \leqslant K \leqslant 2^{P-T}-1$. When the amplitudes $n, d, p, q$ for the $T$ iteration are formed by combining pairs of the previously obtained amplitudes $n, d, p, q$ for the $T-1$ iteration, as in Eqs. (15a)-(15d), the ratio $G_{n}\left(X_{j-1, j}\right) / G_{n}\left(Y_{j, j+1}\right)$ is calculated for

$$
j=2^{T-1}+1+K\left(2^{T}\right)
$$

The integers $K$ span the range from the sphere's core to its surface.

\section{RESULTS}

\section{A. Intensity as a Function of Scattering Angle}

This subsection contains a catalog of the various predicted phenomena when the scattered intensity is plotted as a function of scattering angle. Figure 1 shows $I(\theta)$ for a Luneburg lens with $f=1.0$ and radius $a=8.117 \mu \mathrm{m}$ and with the wavelength of the incident light $\lambda=0.51 \mu \mathrm{m}$. This corresponds to the size parameter $k a=100.0$. The Luneburg lens was divided into $M=2^{7}=128$ layers of width $\Delta=0.0634 \mu \mathrm{m}$, and the parallel iteration procedure was used. Since $\Delta \ll \lambda$, the sphere is considered finely stratified, and subdividing the sphere further revealed no additional structure of $I(\theta)$. The intensity computed for $k a=60.0$ was found to be identical to that of [8], where the progressive iteration procedure was used. The scattered intensity for the TM polarization is seen in Fig. 1 to be almost identical to that for the TE polarization. This is not surprising for the following reason. In ray theory, the

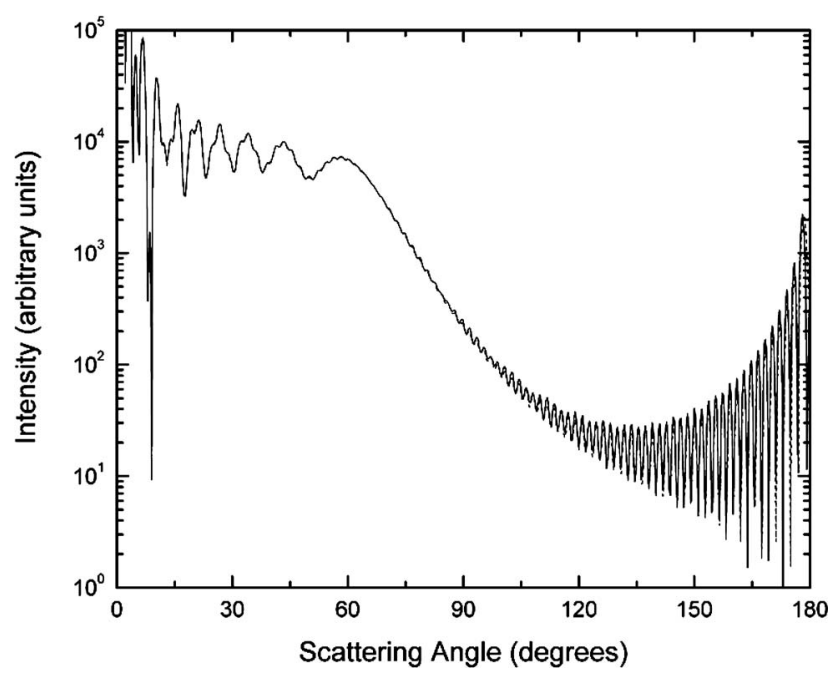

Fig. 1. TE (solid curve) and TM (dashed curve) intensity as a function of the scattering angle $\theta$ for a Luneburg lens with $f$ $=1.0, a=8.117 \mu \mathrm{m}$, and $\lambda=0.51 \mu \mathrm{m}$ computed for an $M=128$ layer sphere using the parallel iteration procedure. 
greatest difference between TE and TM scattering at an interface occurs in the vicinity of the Brewster angle where all of the TM light is transmitted and none is reflected. Two well-known examples of this for scattering of a plane wave by a homogeneous water sphere with $n$ $=1.333$ are as follows. TM external reflection vanishes when $\theta=73.8^{\circ}$, and TM internal reflection vanishes when the angle of incidence on the sphere surface from the inside is $\theta_{t}=36.9^{\circ}$, corresponding to an incident ray impact parameter of $\beta=52.9^{\circ}$. Since this is close to the Descartes impact parameter of the first-order rainbow, $\beta_{R}=59.4^{\circ}$, the first-order rainbow of a homogeneous sphere is dominantly TE polarized. For scattering by a modified Luneburg lens in the finely stratified model, negligible light is reflected at each interface regardless of the angle of incidence, except at near grazing incidence, since the refractive index difference between two adjacent layers is small. Thus the Brewster angle occurs when the angle of incidence on a layer is near $45^{\circ}$. This $45^{\circ}$ incidence condition giving total transmission of the TM ray occurs, at most, twice on a curved ray trajectory inside the sphere, and transmission is otherwise expected to be near total for both polarizations at every other point. Thus TE and TM scattering should be quite similar. For $f=1.1$ and 0.9 , the scattered intensity for the TE and TM polarizations was also computed and found to be nearly identical.

As was discussed in [2], the abrupt transition of the scattered intensity from the ray theory illuminated region to the shadowed region is smoothed in wave theory. The physical optics model predicts that in the vicinity of the weak caustic corresponding to the orbiting ray, the scattered intensity is

$$
I(\theta) \propto\left\{F(\infty)-F\left[\Delta(k a / \pi)^{1 / 2}\right]\right\}^{2},
$$

where

$$
F(w)=\int_{0}^{w} \mathrm{~d} v \exp \left(i \pi v^{2} / 2\right)
$$

and

$$
\theta=(\pi / 2)+\Delta .
$$

Equation (28) is identical to a Fresnel straight-edge pattern and appears prominently in Fig. 1. This is only an approximation, however. For $k a=100$, Eq. (28) predicts that the main peak of the Fresnel straight-edge pattern should occur at $\theta=77.2^{\circ}$, whereas it is observed to occur at $\theta=58.2^{\circ}$ in Fig. 1. Physically, the transition is due to radiation shed by the orbiting ray. For $\theta<58.2^{\circ}$ the shed radiation interferes with rays transmitted through the sphere, producing the oscillations of the Fresnel straightedge pattern. The fine oscillations in Fig. 1 for $\theta>100^{\circ}$ are the interference of the shed radiation of the counterpropagating orbiting rays incident at the top and bottom of the sphere. The path length difference on the surface of the sphere of the two counterpropagating orbiting rays should produce an interference pattern that is independent of $\theta$ and has a periodicity of $\Delta \theta=\pi / k a=1.80^{\circ} /$ cycle. The oscillations in Fig. 1 for $\theta>100^{\circ}$ are in good agreement with this prediction, having the periodicity $\Delta \theta$ $=1.82^{\circ} /$ cycle.
A broad glory enhancement of the scattered intensity is evident for $\theta \approx 180^{\circ}$. For $\theta<60^{\circ}$, the fine oscillations superimposed on the coarser Fresnel straight-edge oscillations are the interference of the transmitted light with the near-forward direction diffracted light. For $\theta<9^{\circ}$ diffraction dominates over transmission. For $\theta \approx 9^{\circ}$ the diffracted and transmitted light of Eq. (18) of [1] have roughly the same magnitude and destructively interfere, producing the large dip in the scattered intensity in Fig. 1. For $\theta>9^{\circ}$ transmission dominates over diffraction, and the transmission-diffraction interference becomes progressively weaker as a function of $\theta$.

Figure 2 compares the TE intensity as a function of $\theta$ for $f=1.1,1.0$, and $f=0.9$, respectively, for $a=8.117 \mu \mathrm{m}$, $\lambda=0.51 \mu \mathrm{m}$, and $M=128$. For $f=1.1$ the predicted Descartes rainbow angle of $\theta_{R}=55.74^{\circ}$ of Eq. (39) of [1] qualitatively agrees with Fig. 2 . The rainbow supernumerary structure is evident for $10^{\circ}<\theta<50^{\circ}$. For $\theta<10^{\circ}$ the supernumerary structure interferes with and merges into the near-forward diffractive structure. The rainbow's complex ray is evident for $\theta>60^{\circ}$. The small amplitude oscillatory structure observed for $\theta>80^{\circ}$ is due to interference of radiation of the complex ray of the rainbow formed from light incident on the top half of the sphere that has damped for less than $180^{\circ}$ and weaker counterpropagating radiation of the complex ray of the rainbow formed from light incident on the bottom half of the sphere that has damped for greater than $180^{\circ}$. The TM intensity was also calculated for $f=1.1$ and $f=0.9$ and was found to be nearly identical to the TE intensity of Fig. 2. In particular, the transmission rainbow for $f=1.1$ is virtually identical for the TE and TM polarizations.

The main rainbow peak of Fig. 2 for $f=1.1$ decreases into the main peak of the Fresnel straight-edge intensity pattern of the orbiting ray when $f=1.0$. The rainbow supernumeraries evolve into the oscillatory structure of the Fresnel straight-edge pattern, and the rainbow's complex ray grows into radiation continuously shed by the orbiting ray. Since the scattering angle of the orbiting ray in Fig. 1

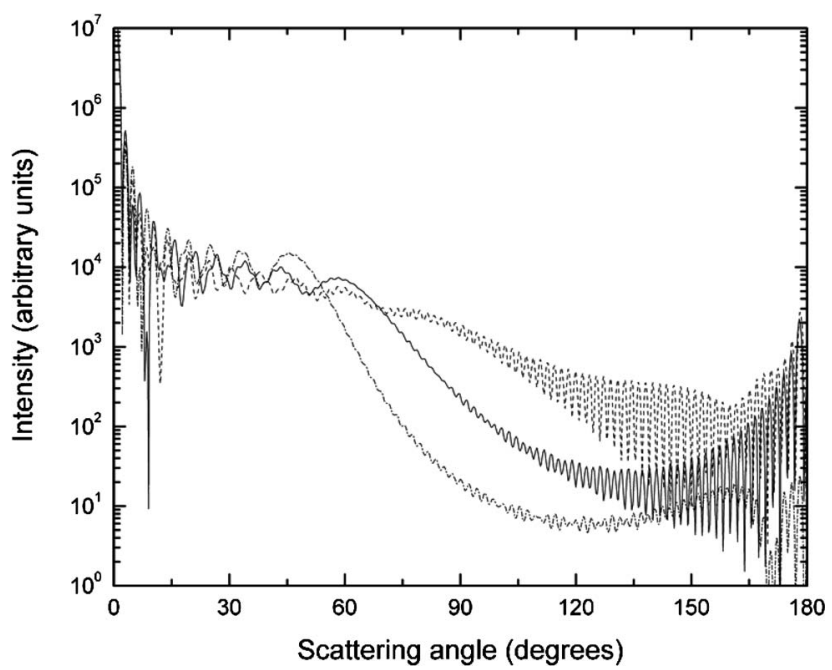

Fig. 2. TE intensity as a function of the scattering angle $\theta$ for a modified Luneburg lens with $f=0.9$ (dashed curve), $f=1.0$ (solid curve), and $f=1.1$ (dotted-dashed curve) for $a=8.117 \mu \mathrm{m}$ and $\lambda$ $=0.51 \mu \mathrm{m}$ computed for an $M=128$ layer sphere using the parallel iteration procedure. 
is larger (i.e., $\theta=90^{\circ}$ in ray theory) than that of the rainbow ray in Fig. 2 (i.e., $\theta_{R}=55.74^{\circ}$ ) and the radiation shed by the orbiting ray for $f=1.0$ falls off more slowly than does the rainbow's complex ray for $f=1.1$, the interference pattern in Fig. 1 for $\theta>90^{\circ}$ has both a larger baseline and a larger modulation depth than does the corresponding interference pattern for $f=1.1$ in Fig. 2 .

For $f=0.9$ in Fig. 2, the orbiting peak has largely dissipated and the falloff of the intensity for $\theta>90^{\circ}$ is slower than for $f=1.0$ in Fig. 1. Although rays are now transmitted through the sphere for this angular range, a large amplitude interference pattern again having the periodicity $\Delta \theta=1.82^{\circ} /$ cycle occurs in Fig. 2, signaling the presence of at least one more ray in this angular region. The origin of this additional ray can be understood as follows. For $f<1$, the grazing incidence ray of Fig. 2(b) of [1] exits the sphere at $\theta=180^{\circ}$ tangent to its surface. This tangency at only one point at the exit likely causes electromagnetic surface waves [16] for $\theta$ larger than $180^{\circ}$ that contribute to the interference pattern. This interpretation is qualitatively supported by the fact that a geometrical ray and a counterpropagating surface wave should have nearly the same amplitude for $\theta$ near $180^{\circ}$ and should produce the type of large modulation depth interference pattern observed in Fig. 2. Finaly, the glory enhancement is again evident in Fig. 2 for $\theta \approx 180^{\circ}$, and the transmitted light again interferes with diffracted light at small angles.

\section{B. TE and TM Resonances}

Figures 3(a) and 3(b) show the scattering efficiency as a function of sphere radius for $f=0.9, \lambda=0.51 \mu \mathrm{m}$, and $M$ $=128$. The TM efficiency has been vertically offset from the TE efficiency by 0.10 for clarity. The optical theorem [17] relates the scattering efficiency to the total scattering amplitude in the forward direction, which here is composed of diffraction plus transmission. The coarse oscillations in the scattering efficiency in Fig. 3(a) are due to diffraction-transmission interference. From Eq. 33 of [1] the ray transmitted through a modified Luneburg lens in the forward direction acquires a phase of

$$
\varphi_{\text {trans }}=k a+k a\left[\left(f^{2}+1\right) / f\right] \arcsin \left[\left(f^{2}+1\right)^{-1 / 2}\right]-\pi,
$$

and the diffracted ray in the forward direction acquires a phase of [18]

$$
\varphi_{\text {diff }}=2 k a+\pi / 2 .
$$

Transmission and diffraction constructively interfere in the forward direction when

$$
\varphi_{\text {trans }}-\varphi_{\text {diff }}=2 \pi P,
$$

where $P$ is an integer. For $f=0.9$, the sphere radii for constructive interference predicted from Eqs. (31)-(33) are $a=4.279 \mu \mathrm{m}, \quad 5.023 \mu \mathrm{m}, \quad 5.768 \mu \mathrm{m}, \quad 6.512 \mu \mathrm{m}, \quad$ and $7.256 \mu \mathrm{m}$, which closely agree with the results of Fig. 3(a). Equations (31)-(33) were found to also accurately describe the coarse oscillations in the scattering efficiency for $f=1.0$ and $f=1.1$, which was free of MDR structure.

The sequence of small peaks superimposed on the diffraction-transmission interference in Fig. 3(a) and which are shown in more detail in Fig. 3(b) are the first radial order (i.e., $S=0$ ) MDRs. The size parameter of

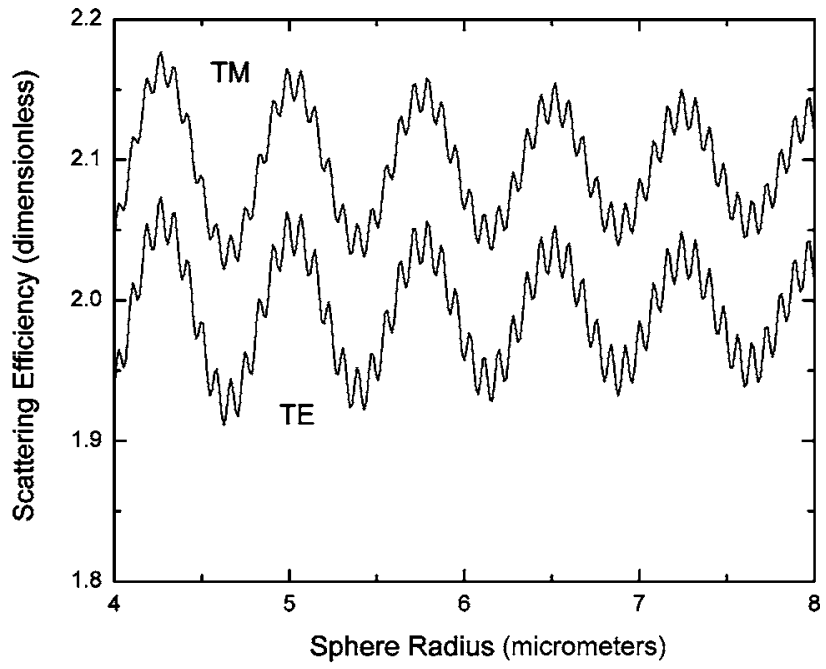

(a)

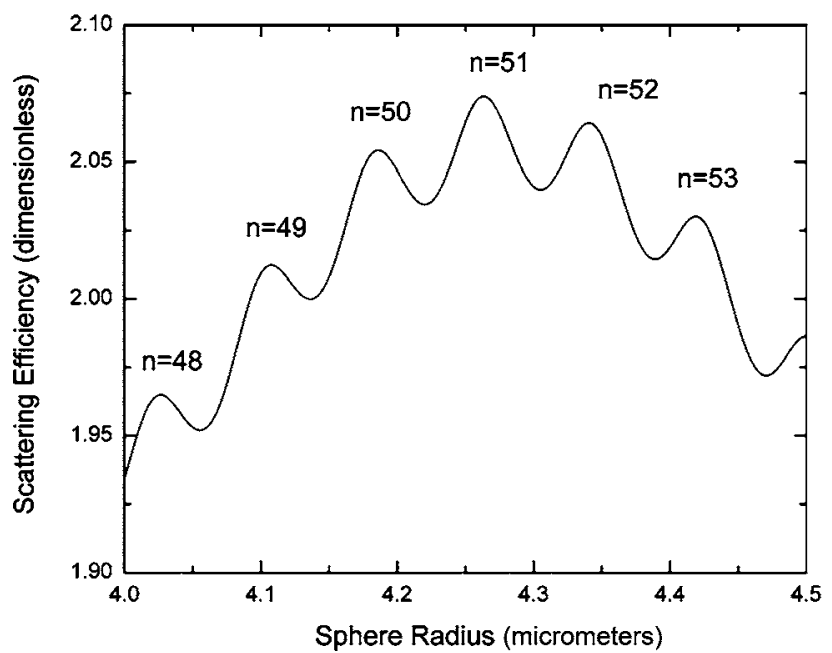

(b)

Fig. 3. (a) Scattered efficiency $\varepsilon$ as a function of sphere radius for a modified Luneburg lens with $f=0.9$ and $\lambda=0.51 \mu \mathrm{m}$. The efficiency for the TM polarization has been vertically offset by $\Delta \varepsilon$ $=0.1$ for clarity. (b) TE scattered efficiency $\varepsilon$ for a smaller range of sphere radii illustrating the $S=0$ resonances in the partial waves $n=48$ through $n=53$.

these resonances is given in Table 1 and was numerically determined by locating the value of $k a$ for a given partial wave, where $a_{n}$ or $b_{n}$ took on the maximum value of 1.0 for $N(r)$ real. The full width at half-maximum of the resonances is about $\Delta k a \approx 0.37$, and the uncertainty in the resonance positions due to the numerical search employed is $\Delta k a= \pm 0.006$. It is felt that these few resonances, and the resonances for $f=0.75$ considered below, are representative of resonances of a modified Luneburg lens. An estimate of the resonant size parameter based on the locations of bound states in the locally parabolic effective radial potential inside the sphere is [2]

$$
k a=(n+2 S+3 / 2)\left[2 f /\left(f^{2}+1\right)\right],
$$

where $S=0,1,2,3 \ldots$ is the radial order. Contrary to the MDRs of a homogeneous sphere [19-21], the size parameters of the TE and TM resonances of a modified Luneburg lens are found to be nearly identical. In Table 1, the 
Table 1. TE and TM Resonant Size Parameters for $f=0.9, \lambda=0.51 \mu \mathrm{m}$, and $M=128$ as a Function of the Partial Wave Number $n^{a}$

\begin{tabular}{llll}
\hline$n$ & $(k a)^{\mathrm{TE}}$ & $(k a)^{\mathrm{TM}}$ & $(k a)^{\mathrm{TE}}$ of Eq. $(34)$ \\
\hline 48 & 49.600 & 49.613 & 49.226 \\
49 & 50.586 & 50.598 & 50.221 \\
50 & 51.584 & 51.584 & 51.215 \\
51 & 52.569 & 52.582 & 52.210 \\
52 & 53.555 & 53.567 & 53.204 \\
53 & 54.553 & 54.553 & 54.199 \\
54 & 55.538 & 55.551 & 55.193 \\
55 & 56.524 & 56.536 & 56.188 \\
\hline
\end{tabular}

${ }^{a}$ The numerical uncertainty in each size parameter is \pm 0.006 . The predicted TE size parameters of Eq. (34) are given in the final column.

size parameters of the TM resonances are larger than the TE resonant size parameters by $\Delta k a=0.009$ on average, whereas for a homogeneous sphere with $n=50, S=1$, and $N=1.47$, the difference is [20] $\Delta k a=0.469$. The differing size parameters of TE and TM resonances of a homogeneous sphere are due to the fact that (i) the interior functions for both polarizations are combinations of RiccatiBessel functions and Riccati-Neumann functions and that (ii) the TE and TM boundary conditions differ. This difference of boundary conditions causes an $S$-radial order MDR (with $S=1,2,3 \ldots$ for a homogeneous sphere) for the TE polarization to have $S$ humps inside the sphere, whereas the TM resonance has $S+1$ humps inside [22]. On the other hand, for a modified Luneburg lens, (i) the interior functions $F_{n}(k r)$ and $G_{n}(k r)$ of [2] for the TE and TM polarizations differ, but (ii) since the interior functions are functions of $k r$ rather than $N k r$ and $N(a)=1$, the TE and TM boundary conditions to be matched at the sphere surface become identical. Thus one can conclude that differences in boundary conditions are more important than differences in radial functions for producing the differing size parameters of TE and TM resonances. The estimated size parameters of Eq. (34) are on average $\Delta k a=0.357$ larger than the values in Table 1 . But the average estimated distance between that adjacent TE resonances of Eq. (34) is $\Delta k a=0.994$, which agrees well with the computed average distance of $\Delta k a=0.989$ between the $\mathrm{TE}$ resonances in Table 1 . These comparisons indicate that Eq. (34) is a reasonably accurate first-order approximation of the positions of the resonances of a modified Luneburg lens.

Perhaps the most novel feature of the modified Luneburg lens MDRs is their value of

$$
X=n(n+1) /(k a)^{2} .
$$

As was discussed in [2], for $X>1$ a partial wave radial function must tunnel through the centrifugal barrier to get to the interior well and be resonantly captured by it. For a homogeneous sphere, this is exactly what occurs and all resonances have $X>1$ (see, for example, Tables 1 and 2 of [20]). But for a modified Luneburg lens, the MDRs of Table 1 have an average $X$ value of 0.960 ; i.e., the MDR is formed before the centrifugal barrier has grown high enough to require tunneling. The fact that $X$ $<1$ for these resonances can be qualitatively understood using the effective potential analysis of [2]. The effective radial potential for the $\mathrm{TE}$ resonance of $f=0.9$ and $n=50$ at $k a=51.584$ is shown in Fig. 4. In the language of quantum mechanics, an attractive potential supports a number of bound states whose energies are various heights above the bottom of the potential well. The ground state energy of a harmonic oscillator lies quite close to the bottom of its parabolic well and is significantly closer to the bottom than is the ground state of either an infinite square well or an Airy well. When the effective potential approach is applied to light scattering, the partial wave number is considered fixed and the incident partial wave has a fixed energy. As the size parameter of the scatterer is varied, the effective potential and its bound states move up or down in concert. In the approximation considered in [2], when the energy of one of the bound states coincides with the fixed energy of the partial wave, an MDR is formed. Such is the case in Fig. 4, the $S=0$ ground state energy of the locally parabolic potential well inside the sphere coincides with the fixed energy of the $n=50$ partial wave. In the trajectory model of ray theory, the corresponding ray is incident on the sphere near its edge and penetrates into it. As it enters the sphere its radial velocity immediately slows and remains relatively small from the sphere surface to the classical turning point at $r / a$ $=0.835$ and then back to the surface again. The ray spends slightly more time inside the sphere than it would if the sphere were slightly larger or smaller. The enhanced scattering of an MDR is associated with the longer interaction time. This resonance occurs even though there is no centrifugal barrier straddling the sphere surface, holding the partial wave in the interior well.

Figures 5(a) and 5(b) show the TE scattering efficiency as a function of the sphere size parameter for $f=0.75, \lambda$ $=0.51 \mu \mathrm{m}$, and $M=128$. In addition to a spectrum of $S$ $=0$ resonances, there is now a spectrum of very weak $S$ $=1$ resonances. Again the size parameters of the TE and TM resonances nearly coincide. For example, for the partial wave $n=50$, the TE resonance with $S=0$ occurs at $k a=49.403$, giving $X=1.045$. The $S=1$ resonance occurs at

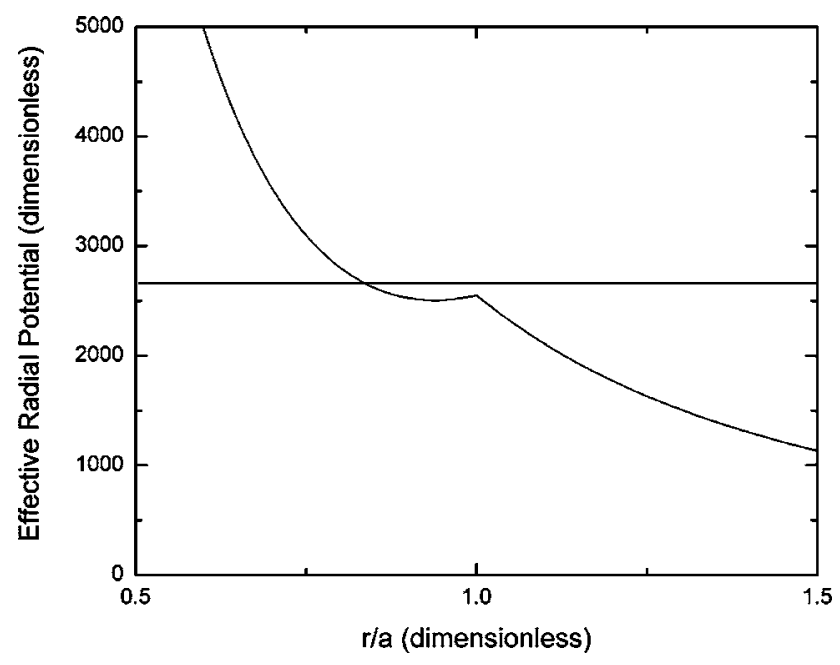

Fig. 4. Effective radial potential of the partial wave $n=50$ as a function of $r / a$ for $k a=51.584$ corresponding to the $S=0$ resonance. The effective energy of this size parameter is denoted by the horizontal line $U_{\text {eff }}=2661$. 


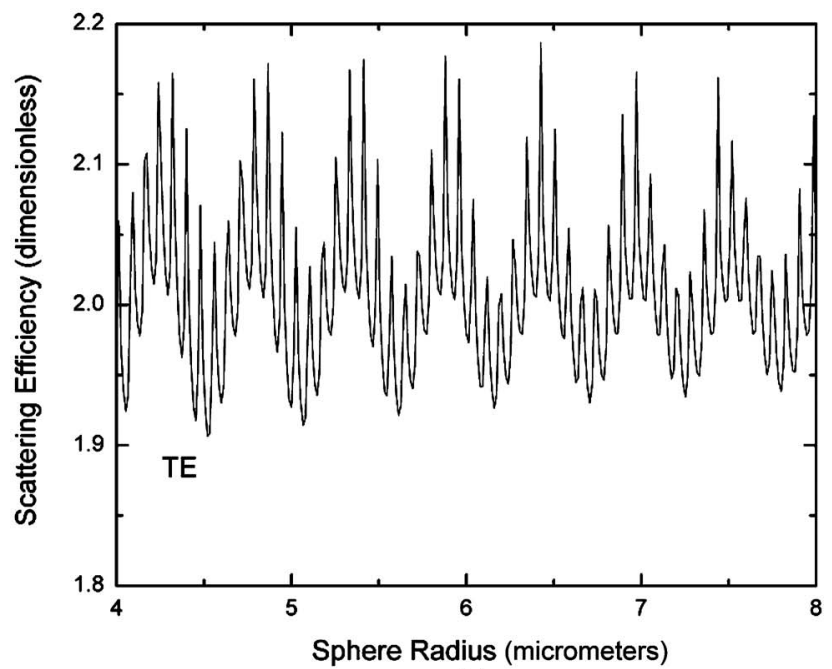

(a)

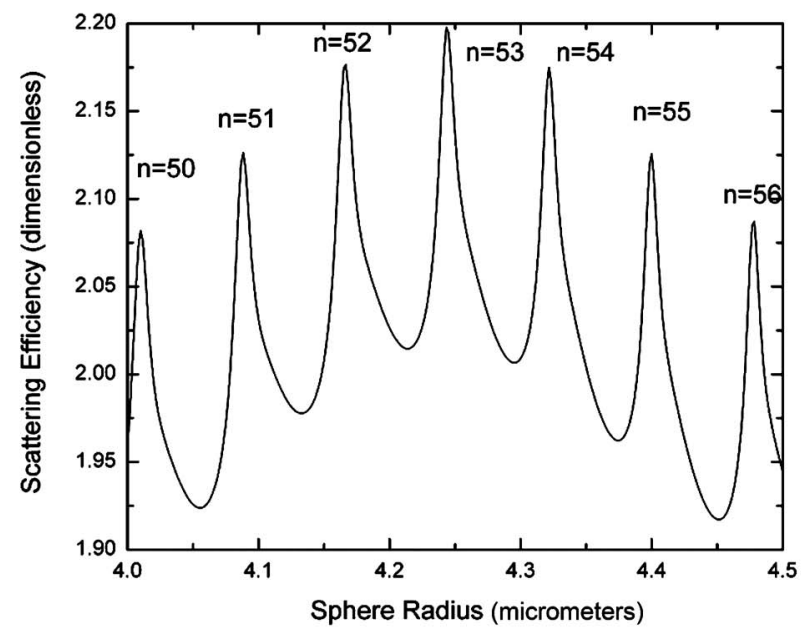

(b)

Fig. 5. (a) TE scattered efficiency $\varepsilon$ as a function of sphere radius for a modified Luneburg lens with $f=0.75$ and $\lambda=0.51 \mu \mathrm{m}$. (b) TE scattered efficiency $\varepsilon$ for a smaller range of sphere radii illustrating the $S=0$ resonances in the partial waves $n=50$ through $n=56$.

$k a=51.510$, giving $X=0.961$. For the TM polarization, the resonances occur at $k a=49.415$ and $k a=51.522$. The full width at half-maximum of the $S=0$ resonances has narrowed to $\Delta k a \approx 0.25$, and the uncertainty in the resonance positions due to the numerical search employed is again $\Delta k a= \pm 0.006$. The $S=0$ resonances appear prominently in Fig. 5(b), and each of the much weaker $S=1$ resonances appear as a shoulder on the right side of each $S=0$ peak. The effective potential wells for the two TE resonances are shown in Figs. 6(a) and 6(b). The $S=0$ ground state of the locally parabolic well now occurs when the well and its bound states have been raised upward in Fig. 6(a) so that a small centrifugal barrier is present for 0.965 $\leqslant r / a \leqslant 1.022$. It is suggestive to attribute the narrower width of the $S=0$ resonances here to the presence of the weak centrifugal barrier for $f=0.75$ that was absent in Fig. 4 for $f=0.9$. The $S=1$ first excited state occurs higher in the well, so the well and its bound states must be lowered in Fig. 6(b) in order to make the first excited state coincide with the fixed energy of the incident partial

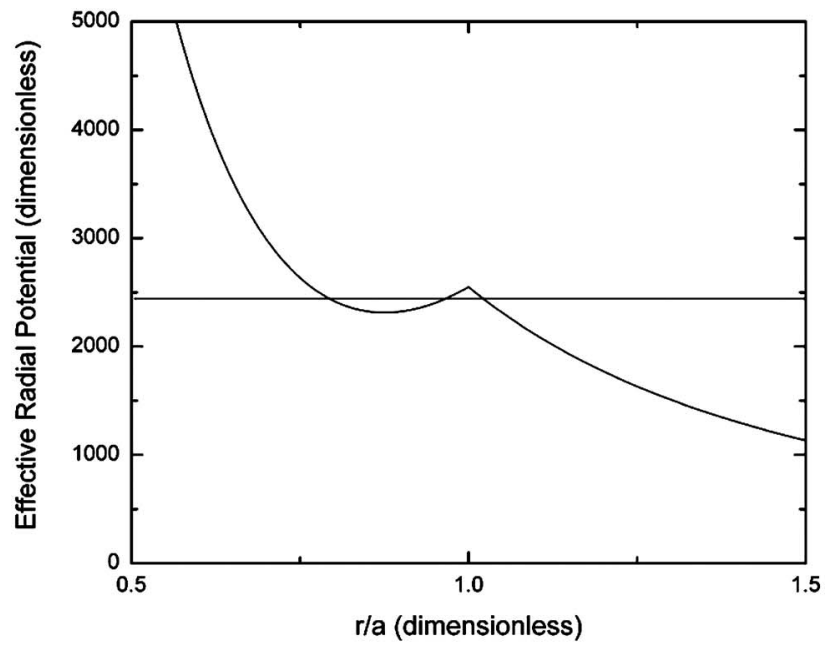

(a)

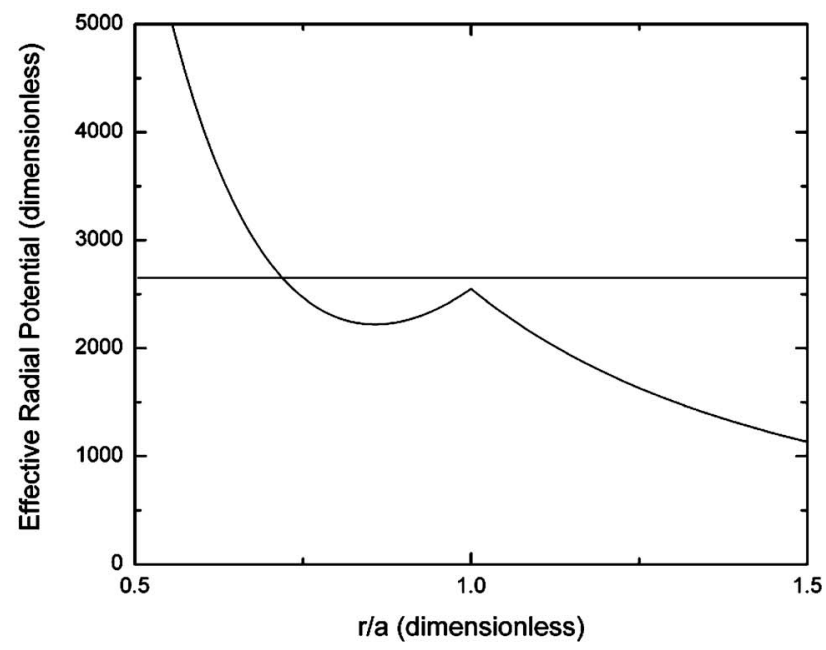

(b)

Fig. 6. Effective radial potential of the partial wave $n=50$ as a function of $r / a$ for (a) $k a=49.403$ corresponding to the $S=0$ resonance and (b) $k a=51.510$ corresponding to the $S=1$ resonance. The effective energy of this size parameters is denoted by the horizontal line $U_{\text {eff }}=2441$ in (a) and $U_{\text {eff }}=2653$ in (b).

wave. As the potential well is lowered, the peak of the centrifugal barrier now falls below the energy of the incident partial wave and no tunneling occurs. The size parameters of the TE resonances estimated from Eq. (34) are $k a=49.440$ for $S=0$, which is higher than the observed TE size parameter by $\Delta k a=0.037$, and $k a=51.360$ for $S=1$, which is lower than the observed size parameter by $\Delta k a=0.150$. Equation (34) is again seen to be reasonably accurate, with its accuracy increasing as the radial order decreases, where the MDR is trapped in a deeper interior well behind a higher centrifugal barrier. The same trend is followed by the first-order estimate of the resonant size parameters of a homogeneous sphere [20]. The widths of the Luneburg lens MDRs examined here are orders of magnitude larger than the widths of the homogeneous sphere MDRs. For a homogeneous sphere, three radial orders of resonances occur [20] for $n \approx 50$. For $S=1$, the full width at half-maximum is $\Delta k a \approx 10^{-6}$, for $S=2$ it is $\Delta k a \approx 10^{-4}$, and for $S=3$ it is $\Delta k a \approx 10^{-2}$. It is likely that the cause of the orders-of-magnitude difference 
in the width of Luneburg lens MDRs and homogeneous sphere MDRs results from the great difference between the spheres' effective radial potentials. A modified Luneburg lens has a locally parabolic effective potential, either without a centrifugal barrier or with only a minimal one. The Airy effective potential of a homogeneous sphere has a much larger centrifugal barrier adjacent to a refractive index discontinuity. It is known that as a homogeneous sphere acquires an imaginary part to its refractive index, the width of the resonances increases. Similarly, it would be of interest to study the properties of MDRs for a number of very different effective radial potentials in order to determine how the potential shape affects the resonances' properties.

\section{CONCLUSIONS}

Although electromagnetic scattering of a plane wave by a homogeneous sphere has been studied extensively for the last century, scattering by more complicated objects such as spheroids, homogeneous spheres having a number of interior inclusions, and radially inhomogeneous spheres have received less study. A question of great general interest is the following. Putting aside the question of spheroids and homogeneous spheres with inclusions, does scattering by a sphere with a radially inhomogeneous refractive index provide only a perturbation on what one sees for scattering by a homogeneous sphere, or do new scattering phenomena now make their presence known? The characterization [23-25] of droplets of volatile liquids having evaporation at the surface by various optical techniques assumes the perturbation point of view. If a droplet's refractive index rapidly decreases near the surface, the one-internal-reflection rainbow continues to exist in its usual form. But the rainbow scattering angle and supernumerary periodicity change in response to the refractive index decrease near the surface. Similarly, MDRs continue to exist in their usual form, but the resonant size parameter and width change their values slightly [26,27]. Measurement of the rainbow or MDR shifts can be used as a diagnostic to characterize various physical properties of the droplet being studied. But if the droplet shape or refractive index variation becomes large enough, new phenomena occur as well. As an example for the case of shape perturbations, if a homogeneous sphere is distorted into a spheroid and the aspect ratio becomes large enough, the rainbow evolves into a hyperbolic umbilic caustic [28]. Similarly, the axial glory caustic evolves into an astroid as the spherical symmetry of the scatterer is broken [29]. Also, an MDR splits into a family of resonances with differing azimuthal mode numbers [30]. For a sphere with certain radially inhomogeneous refractive index profiles, two or more one-internal-reflection rainbows can occur [31], and the structure of the glory can become more complicated [32].

This set of three papers addresses the above-posed question for the refractive index profile of a modified Luneburg lens. Many of the phenomena of scattering by a homogeneous sphere continue as before. But a number of new effects occur as well. Since $N(r)=1$ inside the sphere as $r \rightarrow a$, internal and external reflection at the surface are minimal at best. The only way to attribute part of the scattering to internal and external reflection is to redefine these processes from a multiple-scattering point of view, using one of the forms of the multilayer sphere Debye series. A transmission rainbow now occurs virtually identically for both the TE and TM polarizations, and all of its details are, surprisingly, exactly soluble in ray theory. One can come very close to the conditions for classical orbiting for $f=1$, and with a more complicated refractive index profile one could exactly achieve the orbiting condition. Finally, MDRs can occur without having a refractive index discontinuity at the sphere surface or an external centrifugal barrier to hold them in. The parabolic variation of the effective radial potential due to the Luneburg lens's special $N(r)$ does the job itself. These effects were identified and analyzed in this set of papers in order to further understand and appreciate the richness of phenomena that occur for electromagnetic scattering by an object as geometrically simple as a sphere when its refractive index profile assumes some degree of complexity. Another analytically soluble refractive index profile is discussed in [33].

A further generalization of the Luneburg lens is the refractive index profile

$$
N(r)=\left\{\left[\left(f^{2}+1\right) / f^{2}\right]-\left(g / f^{2}\right)(r / a)^{2}\right\}^{1 / 2}
$$

for $r \leqslant a$ for the two-parameter family $f$ and $g$. The TE partial wave scalar radiation potential is still a Whittaker function, since the radial dependence of $N^{2}(r)$ is still $r^{2}$. For $g=1$, this new profile reduces to the modified Luneburg lens studied in this series of papers. For $g \neq 1$ the effective potential is discontinuous at $r=a$ causing both internal and external reflection at the surface. For $g<1$, the centrifugal barrier outside the sphere is raised with respect to the value of the radial potential inside. The resulting sphere may give rise to a much narrower set of MDRs, since the centrifugal barrier is now more prominent. For $g>1$, the effective potential inside the sphere is raised with respect to its value outside. This can lead to a centrifugal barrier lying totally inside the sphere with a locally parabolic well lying further inside. It would be of interest to determine whether additional novel scattering phenomena occur if the modified Luneburg lens of Eq. (2) is itself further modified, as in Eq. (36).

\section{REFERENCES}

1. J. A. Lock, "Scattering of an electromagnetic plane wave by a Luneburg lens. I. Ray theory," J. Opt. Soc. Am. A 25, 2971-2979 (2008).

2. J. A. Lock, "Scattering of an electromagnetic plane wave by a Luneburg lens. II. Wave theory," J. Opt. Soc. Am. A 25, 2980-2990 (2008).

3. O. B. Toon and T. P. Ackerman, "Algorithms for the calculation of scattering by stratified spheres," Appl. Opt. 20, 3657-3660 (1981).

4. R. Bhandari, "Scattering coefficients for a multilayered sphere: analytic expressions and algorithms," Appl. Opt. 24, 1960-1967 (1985).

5. D. W. Mackowski, R. A. Altenkirch, and M. P. Menguc, "Internal absorption cross sections in a stratified sphere," Appl. Opt. 29, 1551-1559 (1990).

6. Z. S. Wu and Y. P. Wang, "Electromagnetic scattering for a multilayered sphere: recursive algorithms," Radio Sci. 26, 1393-1401 (1991). 
7. L. Kai and P. Massoli, "Scattering of electromagnetic-plane waves by radially inhomogeneous spheres: a finely stratified sphere model," Appl. Opt. 33, 501-511 (1994).

8. B. R. Johnson, "Light scattering by a multilayer sphere," Appl. Opt. 35, 3286-3296 (1996).

9. Z. S. Wu, L. X. Guo, K. F. Ren, G. Gouesbet, and G. Grehan, "Improved algorithm for electromagnetic scattering of plane waves and shaped beams by multilayered spheres," Appl. Opt. 36, 5188-5198 (1997).

10. J. A. Lock, "Debye series analysis of scattering of a plane wave by a spherical Bragg grating," Appl. Opt. 44, 5594-5603 (2005).

11. R. C. Gonzalez, Digital Image Processing, 3rd ed. (Pearson/ Prentice Hall, 2008), pp. 299-302.

12. H. C. Van de Hulst, Light Scattering by Small Particles (Dover, 1981), pp. 124-125.

13. M. Abramowitz and I. A. Stegun, eds., Handbook of Mathematical Functions (National Bureau of Standards, 1964), p. 365, Eq. (9.3.1); p. 437, Eq. (10.1.1)

14. W. J. Wiscombe, "Improved Mie scattering algorithms," Appl. Opt. 19, 1505-1509 (1980).

15. C. F. Bohren and D. R. Huffman, Absorption and Scattering of Light by Small Particles (Wiley-Interscience, 1983), pp. 127,128 , and 478 .

16. H. M. Nussenzveig, "High-frequency scattering by a transparent sphere. I. Direct reflection and transmission," J. Math. Phys. 10, 82-124 (1969).

17. H. C. Van de Hulst, Light Scattering by Small Particles (Dover, 1981), pp. 176-178.

18. H. C. Van de Hulst, Light Scattering by Small Particles (Dover, 1981), pp. 209-210.

19. P. Chylek, "Partial-wave resonances and the ripple structure in the Mie normalized extinction cross section," J. Opt. Soc. Am. 66, 285-287 (1976).

20. P. Chylek, J. T. Kiehl, and M. K. W. Ko, "Optical levitation and partial-wave resonances," Phys. Rev. A 18, 2229-2233 (1978)

21. C. C. Lam, P. T. Leung, and K. Young, "Explicit asymptotic formulas for the positions, widths, and strengths of resonances in Mie scattering," J. Opt. Soc. Am. B 9, 1585-1592 (1992).

22. P. Chylek, J. D. Pendleton, and R. G. Pinnick, "Internal and near-surface scattered field of a spherical particle at resonant conditions," Appl. Opt. 24, 3940-3942 (1985).

23. M. Schneider and E. D. Hirleman, "Influence of internal refractive index gradients on size measurements of spherically symmetric particles by phase Doppler anemometry," Appl. Opt. 33, 2379-2388 (1994).

24. J. P. A. J. van Beeck and M. L. Reithmuller, "Rainbow phenomena applied to the measurement of droplet size and velocity and to the detection of nonsphericity," Appl. Opt. 35, 2259-2266 (1996).

25. P. Massoli, "Rainbow refractometry applied to radially inhomogeneous spheres: the critical case of evaporating droplets," Appl. Opt. 37, 3227-3235 (1998).

26. D. Q. Chowdhury, S. C. Hill, and P. W. Barber, "Morphology-dependent resonances in radially inhomogeneous spheres," J. Opt. Soc. Am. A 8, 1702-1705 (1991).

27. K. M. Lee, P. T. Leung, and K. M. Pang, "Iterative perturbation scheme for morphology-dependent resonances in dielectric spheres,” J. Opt. Soc. Am. A 15, 1383-1393 (1998).

28. P. L. Marston and E. Trinh, "Hyperbolic umbilic diffraction catastrophe and rainbow scattering from spheroidal drops," Nature 312, 529-531 (1984).

29. W. P. Arnott and P. L. Marston, "Unfolding axial caustics of glory scattering with harmonic angular perturbations of toroidal wave fronts," J. Acoust. Soc. Am. 85, 1427-1440 (1989).

30. H. M. Lai, P. T. Leung, K. Young, P. W. Barber, and S. C. Hill, "Time-independent perturbation for leaking electromagnetic modes in open systems with application to resonances in microdroplets," Phys. Rev. A 41, 5187-5198 (1990).

31. J. A. Adam and P. Laven, "Rainbows from inhomogeneous transparent spheres: a ray-theoretic approach," Appl. Opt. 46, 922-929 (2007).

32. C. L. Brockman and N. G. Alexopoulos, "Geometrical optics of inhomogeneous particles; glory ray and the rainbow revisited," Appl. Opt. 16, 166-174 (1977).

33. A. Y. Perelman, "Scattering by particles with radially variable refractive indices," Appl. Opt. 35, 5452-5460 (1996). 